\title{
EVALUACIÓN DE IMPACTO AMBIENTAL DE LA CONSTRUCCIÓN DE OBRAS PROTECTORAS EN MOA, HOLGUÍN
}

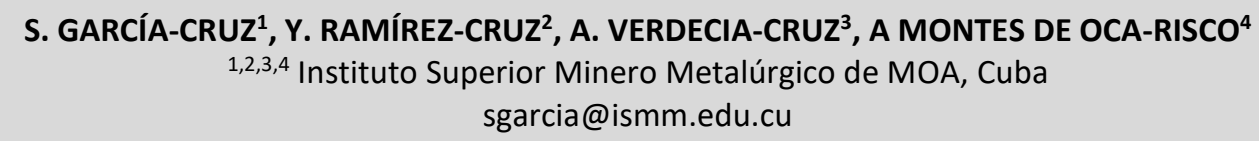 \\ Recepción 09/04/2018 - Aprobacíon 22/11/2018 \\ DOI: $10.15628 /$ holos.2018.7188
}

\section{RESUMEN}

La ejecución y el funcionamiento de las obras protectoras subterráneas, como cualquier obra minera constructiva, no está exenta de generar impactos sobre el medio donde se encuentra situada. Sin embargo la necesidad de construirla no implica necesariamente la degradación indiscriminada del entorno, pues a través de los procedimientos establecidos de Evaluación de Impacto Ambiental y gestión ambiental se pueden minimizar los impactos negativos identificados. Por tal razón se propuso como Objetivo en este trabajo: identificar, caracterizar y evaluar el impacto ambiental producto de la construcción de obras protectoras en el municipio de Moa, con el fin de proponer medidas preventivas y correctoras para compatibilizar la construcción y la preservación del medio natural. En la metodología: se aplicaron métodos científicos que permitieron el análisis de los factores que influyen en la alteración del medio ambiente producto de la construcción de la obra. Alcanzándose como resultado: la matriz de impacto ambiental de la obra objeto de estudio y como conclusión: se propusieron medidas para cada uno de los componentes impactados para mitigar y corregir sus afectaciones.

PALABRAS-CLAVE: Evaluación de Impacto Ambiental, Obras protectoras, obras subterráneas.

\section{EVALUATION OF ENVIRONMENTAL IMPACT OF THE CONSTRUCTION OF PROTECTING BUILDING WORK IN MOA, HOLGUÍN}

\begin{abstract}
The execution and operation of the underground protective works, like any constructive mining work, is not exempt from generating impacts on the environment where it is located. However, the need to build it does not necessarily imply the indiscriminate degradation of the environment, because through the established procedures of Environmental Impact Assessment and environmental management the negative impacts identified can be minimized. For this reason, it was proposed as Objective in this work: to identify, characterize and evaluate the environmental impact resulting from the construction of protective
\end{abstract}

works in the municipality of Moa, with the purpose of proposing preventive and corrective measures to make compatible the construction and the preservation of the environment natural. In the methodology: scientific methods were applied that allowed the analysis of the factors that influence the alteration of the environment resulting from the construction of the work. Reached as a result: the environmental impact matrix of the work under study and in conclusion: measures were proposed for each of the components impacted to mitigate and correct their effects.

KEYWORDS: Environmental Impact Assessment, Protective works, underground works. 


\section{INTRODUCCIÓN}

A partir de la década de 1970 se aceleró la conciencia ecológica y la sociedad comenzó a entender que el origen de los conflictos ambientales se encontraba en las estructuras económicas y productivas de la economía, dado en que los principales problemas que aquejan al medio ambiente, tienen su origen en los procesos productivos mal planificados y gestionados. Es precisamente mediante la transformación de tales sistemas como se podía acceder a una mejora integral de éste.

El estado actual de la población humana en crecimiento explosivo y con necesidades en constante aumento demanda con urgencia la protección de los ecosistemas urbanos lo que implica un uso sostenible de los mismos. Para ello, es menester que este uso se corresponda con las verdaderas necesidades humanas de las presentes generaciones, como condición para salvaguardar la satisfacción futura.

En la actualidad el hombre es considerado el principal responsable de las diferentes acciones agresivas que motivaron las transformaciones en la naturaleza, por lo que se está afectando su equilibrio ecológico y el límite de auto restauración o resiliencia de los ecosistemas. Es por ello que debemos compatibilizar las acciones tecnológicas, científicas, económicas y socioculturales en el entorno natural García (2010).

Pero todas las influencias negativas de las acciones del hombre sobre la naturaleza resultan insuficientes, comparadas con la destrucción que puede ocasionar una guerra. No solo los conflictos armados generan degradación ambiental. La industria de armamento e incluso la preparación y el entrenamiento de las tropas, traen consigo también el deterioro del medio ambiente.

Como resultado de estos peligros, Cuba se ha visto obligada a mantener una elevada preparación defensiva de todo el pueblo para afrontar cualquier tipo de lucha armada y en ese contexto, la construcción de obras protectoras se convierte en una tarea priorizada.

En los obras protectoras del municipio se han desarrollado importantes trabajos, dirigidos al mejoramiento de las condiciones constructivas y de estabilidad de los mismos, pero no ha formado parte de esas investigaciones lo referente a las cuestiones ambientales, justificado por el predominio del interés que ocupan las actividades mineras a cielo abierto, sumamente agresivas al medio ambiente y los efectos de los procesos metalúrgicos, constituyendo la situación problémica de esta investigación.

Una aproximación a esta situación problémica demostró la existencia del siguiente Problema de la investigación: La necesidad de realizar una evaluación de impacto ambiental originados por la construcción de obras protectoras para minimizar y corregir los impactos negativos sobre el medio ambiente.

Con vista a la solución de este problema se propone como objetivo de la investigación, realizar una evaluación de impacto ambiental originados por la construcción de obras protectoras, con el fin de proponer las medidas preventivas y correctoras que habrán de adoptarse para la minimización y corrección de los impacto negativos sobre el entorno, a fin de compatibilizar la construcción y la preservación del medio natural. 


\section{REVISIÓN BIBLIOGRÁFICA}

\subsection{Antecedentes del uso de las obras protectoras}

Una de las posibles formas de adentrarse en la historia y la cultura de los pueblos es estudiando las construcciones subterráneas. Resulta fascinante adentrarse en ese reflejo de las civilizaciones a partir de la forma en que construían los subterráneos, tomando como referencias sus creencias y mitos en torno a lo profundo, observando el enfoque místico o funcional dado al adentrarse en la tierra.

El hombre primitivo no solo se guarece en la cueva sino que desde ella se proyecta, invoca la caza a través de las pinturas rupestres, primera manifestación artística- mágica del ser humano. Aquí la cueva ya es mucho más que receptáculo pétreo que cobija de una climatología adversa; constituye de alguna manera un vínculo de unión con la vida, con la supervivencia a través de la potencia que irradia del arte parietal.

Desde épocas antiguas, el hombre ha utilizado las obras subterráneas durante las guerras como refugio para salvaguardar sus bienes, familiares, armamento y para combatir directamente desde ahí.

Las obras subterráneas pueden ser de tipos diversos, por sus cometidos y el entorno en que se ubican; así hay urbanos, rurales, de montaña, subacuáticos, de carretera, dependiendo las consideraciones medioambientales de tipo de túnel de que se trate, es decir del entorno en que se ubique, de las ventajas socioeconómicas que reporte y de las características de la obra superficial a la que sustituye.

Resulta impresionante la tremenda perseverancia y el desprecio por el riesgo que mostró el hombre desde sus orígenes de la Historia en sus intentos de perforar la Tierra, partiendo inicialmente sólo de las propias manos y la fuerza bruta y poco a poco, confeccionando herramientas, rudimentarios martillos, picos y cinceles.

En las obras protectoras se protegen cientos y miles de personas fácilmente. Se construyen en tiempo de paz por lo general. Son seis veces más económicos que las Obras Ingenieras a cielo abierto y 90 veces más invulnerables a los medios de destrucción del enemigo. Con los túneles se logra un aumento de la capacidad y disposición combativa, producto de la desconcentración y porque su ubicación se corresponde, en la mayoría de los casos, con alturas dominantes que pasan a ser centros fortificados, desde donde las tropas tienen todas las ventajas para batir al enemigo García (2010).

\subsection{Análisis y Revisión Bibliográfica}

En los últimos años son numerosas las investigaciones realizadas a nivel internacional acerca de la evaluación de impacto ambiental.

Es importante destacar en el sentido teórico metodológico los trabajos realizados por: Leopold et al. (1971) y Sorenssen et al. (1973), proponen diferentes variantes de matrices, en diferentes tipos de proyectos que permite apropiarnos de conocimientos para la selección de la metodología adecuada de identificación de impactos para este tipo de investigación. Por otra parte Cabrera (1987) y Hernández (2003) en investigaciones realizadas acerca de Evaluación de 
Impacto Ambiental", proponen los métodos más conocidos para la identificación y valoración de los impactos ambientales, que tuvimos en cuenta en esta investigación, sobre la base del nivel de intensidad de las evaluaciones, aportando criterios sobre las ventajas y limitaciones para su aplicación en diferentes circunstancias y momentos por los que atraviesan los proyectos a tener en cuenta en cualquier tipo de obra.

Además es meritorio destacar los trabajos realizados por Conesa en (1997) y Gómez (1999), en su manual" Guía Metodológica para la Evaluación de Impactos Ambientales", dos clásicos de este tema, ofrecen procedimientos y métodos de trabajo que permiten realizar un enfoque integral. Además proponen el uso de métodos cualitativos y cuantitativos, las matrices y una tipología de clasificación de 10 clases de impactos. En su matriz de importancia relaciona las acciones, tanto en la fase constructiva como en la fase de funcionamiento del proyecto y los factores del medio que pueden ser afectados por dichas acciones.

Años más tarde también lo hace Sánchez (2001), en su artículo "Evaluación de Impacto Ambiental" en el Segundo Curso Internacional de Aspectos Geológicos de Protección Ambiental, donde utiliza dos enfoques distintos, pero complementarios. El primer enfoque estudia el proceso de Estudio de Impacto Ambiental, o sea, los procedimientos que debe ser ejecutados para identificar, prever y evaluar la importancia de las consecuencias futuras de las decisiones actuales. El segundo enfoque se centra en los métodos y las herramientas de identificación, previsión y evaluación de los impactos ambientales.

Como referencia, para la realización de Estudio de impacto ambiental han sido consultadas las tesis de maestría en la mención Geología Ambiental realizadas en el Instituto Superior Minero Metalúrgico de Moa (ISMM) tales como: Estudio de la Influencia en el Medio Ambiente del Sistema de Generación de la Empresa del Níquel Comandante Ernesto Che Guevara de (Hurtado 2012), Estudio de Impacto Ambiental en Obras subterráneas en funcionamiento del Ejército Oriental" (Suárez 2007), "Estudio de Impacto Ambiental del Proyecto de Explotación del Yacimiento Punta Gorda", de, expone la caracterización del medio ambiente del territorio, y aplica una metodología para evaluar el impacto de las zonas afectadas por la explotación de yacimientos lateríticos (Hernández 2013), Estudio del Impacto Ambiental ocasionado por la Explotación del Yacimiento Fluvial de Arena y Grava Río Nibujón (Aguilera 2003) entre otras.

Se tuvieron en cuanta además las investigaciones realizadas en los túneles, encaminadas por el grupo de construcción subterránea del ISMM, dentro de los que se encuentran Caracterización geomecánica de los macizos rocosos de la Mina Merceditas (Cartaya, 2000) y la Evaluación del método de arranque más eficiente para el laboreo de excavaciones subterráneas en la región oriental (Noa, 2003), entre otras investigaciones.

Todas estas investigaciones y bibliografías consultadas han servido de base para desarrollar la presente investigación. Sin embargo en los trabajos realizados en los túneles populares no han considerado los aspectos medio ambientales pasando esto a un segundo plano hasta la fecha, lo que ha estado motivado por el predominio del interés que ocupa lo relacionado con los efectos de las actividades mineras a cielo abierto, y los procesos metalúrgicos sumamente agresivos al medio ambiente. 


\subsection{Antecedentes de la problemática ambiental de Moa}

Para poder analizar las condiciones ambientales del entorno, que de forma simple podemos definir como crítica, debemos partir del entendimiento de los problemas ambientales del país, los que tienen su origen y dimensión, en las formas inapropiadas en que, por varios siglos, fueron explotados los recursos naturales, las limitaciones e insuficiencias con que Cuba enfrentó el proceso de industrialización, la inadecuada técnica agropecuaria y los impactos ocasionados por la situación social en la etapa pre-revolucionaria.

Pero tampoco podemos obviar que después del Triunfo de la Revolución, aún cuando se ha trabajado para solucionarlos, las limitaciones económicas como país del tercer mundo, agravadas por el bloqueo imperialista, no han permitido revertir en la magnitud necesaria las deficiencias heredadas.

Además, el desarrollo vertiginoso de programas económicos no siempre contó con la solución adecuada respecto al tratamiento de residuales, emplazamientos urbanos y cuidado integral del medio, todo ello influido por una falta de conciencia y educación ambiental en una parte considerable de la población.

Inciden además en la situación cubana los problemas de deterioro a nivel regional o global como por ejemplo los cambios climáticos.

La identificación de los principales problemas del país, jerarquizar su atención y dirigir hacia ellos los principales esfuerzos de la gestión ambiental ha constituido un objetivo básico en los últimos años, cuando en 1992 se adoptaron los acuerdos de Río de Janeiro en la Cumbre de la Tierra, plasmados en la Agenda 21.

Al evaluarse la evolución que ha tenido el medio ambiente cubano, puede afirmarse, que existen avances, dado por las profundas transformaciones económicas y sociales, que han permitido alcanzar importantes metas, que para muchos países resultan aún lejanas.

Entre los principales problemas que afectan al medio ambiente en el entorno moense está la degradación de los suelos. Existen dos causas principales que degradan los suelos, una relacionada con la actividad agrícola y la otra, la minera. Es el segundo grupo de actividades el responsable del impacto al suelo en Moa. Los yacimientos ferroniquelíferos son explotados con el sistema de minas a cielo abierto y ello implica la deforestación y destape de las menas. De esta forma se expone a la acción de las aguas superficiales y en menos grado por el viento, un suelo desprotegido por la pérdida de la cobertura vegetal.

Los materiales así acarreados pasan a formar parte de las corrientes fluviales, depositándose en estas o transportándose hasta el mar donde se acumulan, creando a su vez otros problemas como: contaminación de las aguas alterando su turbidez; del aire, aumentando las partículas en suspensión, colmatando la franja litoral con sedimentos, dentro de lo que se encuentra la dársena del puerto.

Los medios para controlar este fenómeno existen y se aplican en la minería actual, pero no siempre con la inmediatez que se requiere, además de que existen áreas minadas en periodos anteriores a este nuevo enfoque de la sustentabilidad que aún esperan por la acción conservacionista. 
Otro problema ambiental es el deterioro del saneamiento y de las condiciones ambientales en los asentamientos humanos, dado por la contaminación del aire derivada de las emanaciones industriales, dado por la pobre aplicación del conocimiento científico en la prevención de estos problemas, y la violación de la legislación ambiental. A lo anterior se unen las dificultades para acceder a tecnologías de punta y la no disponibilidad, por su elevado costo, de sistemas modernos y eficientes de depuración de las emisiones.

Lo evidente es que pueden encontrarse soluciones, con las mismas herramientas científicas con que se aumenta y se expande la minería y la producción metalúrgica y por supuesto, con los mismos hombres.

La contaminación de las aguas terrestres y marinas, tanto por partículas sólidas como por residuales líquidos es otro de los problemas que se confrontan en el municipio y aun cuando no es muy elevado la incidencia sobre el agua que consume la población, tiene efectos negativos sobre los ecosistemas acuáticos y sus alrededores. Ejemplos en este caso son muchos: albañales que van a ríos, arroyos o al mar, vertidos de la fábrica Pedro Soto en Río Cabaña y de la Ernesto Che Guevara en la presa de cola que filtra hacia el mar y el manto freático.

Además de los altos índices de contaminación en el municipio, dado por los factores antes señalados existen otras fuentes de impacto al medio ambiente como el tratamiento insuficiente de residuales de origen doméstico, los niveles de contaminación acústica en algunas áreas, el impacto sobre el paisaje de la actividad minera e industrial así como la insuficiente calidad y cantidad de las áreas verdes.

Muchos de estos problemas que persisten en el medio tienen explicación, pero también tienen solución. Sabemos por ejemplo que la minería se hace con una concepción mucho más satisfactoria para el cuidado del ambiente, que se protegen las cuencas hidrográficas del deslave de las minas, que se intenta reforestar y se logra en algunos sectores, pero también sabemos que no es suficiente.

En el transcurso de los años de una forma $u$ otra estos problemas han incidido negativamente en la diversidad biológica, pues se ha maltratado el hábitat natural de especies y además, afectan la salud humana.

\section{METOdOLOGÍA}

\subsection{Caracterización del área de estudio}

Ubicación del área de estudio

Moa es una rica región minera situada al noroeste de la provincia Holguín, limita al norte, con el Océano Atlántico, al sur y este con el Municipio de Baracoa y al oeste con el Municipio Sagua de Tánamo (Figura 1). El territorio tiene una extensión de $732,6 \mathrm{Km}^{2}$. Su población asciende a más de 70 mil habitantes. Es un Municipio de alto grado de urbanización y se asienta en dos núcleos urbanos: Moa como una comunidad urbana propiamente dicha, y Punta Gorda García (2010). 


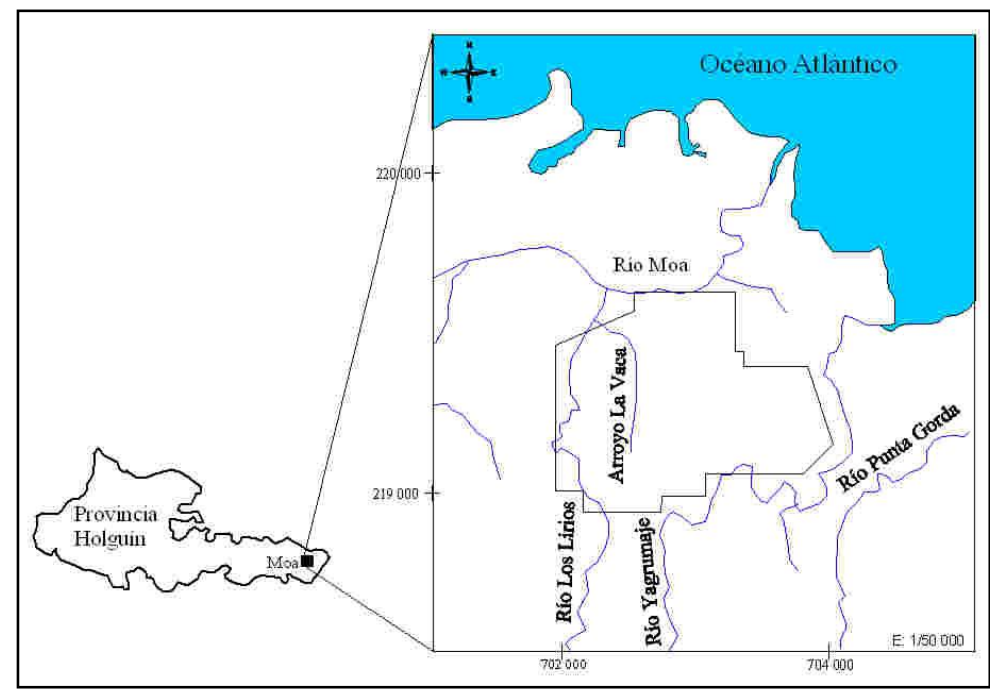

Figura 1: Ubicación del área de estudio. Fuente: Almaguer(2010).

Clima

El clima de la región es tropical, caracterizado por la presencia de dos períodos de lluvia (Mayo-Junio y Octubre-Enero) y dos períodos de seca (Julio-Septiembre y Febrero-Abril). Este clima se encuentra influenciado por la orografía, sirviendo de pantalla a los alisios del NE las barreras montañosas del Grupo Sagua - Baracoa. La zona se encuentra bajo el régimen de brisas y terrales así como de vientos gravitacionales (vientos que descienden siguiendo el curso de los ríos aproximadamente).

Los datos de temperatura fueron tomados de la Estación Hidrometereológica "El Sitio". Según observaciones realizadas en los años 2000-2008, se evidencia que los meses más calurosos son desde julio hasta septiembre, lográndose temperaturas de 27,$1 ; 29,0$ y $30,5{ }^{\circ} \mathrm{C}$ respectivamente, mientras que los más fríos son enero-febrero, con temperaturas promedios de 22,6 y $23,2{ }^{\circ} \mathrm{C}$ respectivamente.

Suelo

Existe variedad de suelos producto al clima, la vegetación y la morfología. En la zona costera baja aparecen Ciénagas con un terreno carmelita grisáceo, muy arcilloso y con un alto contenido de material orgánico. En las riberas de los ríos aparecen suelos aluviales de diferentes tamaño y color. En el territorio predominan los suelos aluviales formados de la meteorización de las rocas serpentinizadas y gabroides. Estos suelos pueden alcanzar potencias considerables de más de $50 \mathrm{~m}$ en bolsones situados en zonas tectónicas. En sentido general, las cortezas más potentes se desarrollan en las partes inferiores de las laderas con pendientes suaves y aplanadas en forma de mesetas. En las cimas más elevadas, los suelos son pardos, violetas, rojos y amarillentos. Estos no fueron sometidos a un proceso de sumersión, lo que puede probarse porque muchas plantas primitivas se conservan, han evolucionado, adaptándose a estos terrenos. La composición química de estos terrenos, ricos en distintos minerales pesados, lo hacen poco fértil, dificultando la alimentación de la población en lo que respecta a los productos del agro. El drenaje superficial y subterráneo en estos suelos son buenos y en ocasiones excesivos, lo que unido a sus características físicos - mecánicas y las intensas precipitaciones y el tectonismo del terreno, dan lugar a la fuerte erosión laminar y en cárcavas. García (2010) 
Flora y Fauna

El municipio de Moa tiene una situación particular, por las características de su vegetación, que ha provocado la evolución de una flora muy típica que comprende el $33 \%$ de todos los endemismos cubanos, compuesta por pinares, pluvisilvas, charrascales y bosques de galerías.

La fauna presenta heterogeneidad y abundancia de especies raras con características peculiares, desde las pequeñas microrrisas hasta el cocosí, además de poder contar con la vistosa cotorra, la cartacuba, el ruiseñor, el catey, el zunzún, el murciélago, el colibrí, entre otros, que corresponden a los grupos característicos de muchos bosques del país. Se reportan como endémicas 104 especies, 17 son exclusivas de Moa y 13 vulnerables a desaparecer.

Al hacer un balance integral de la biodiversidad se reportan un total de 772 especies, de ellos, 317 son taxones infragenéricos endémicos, 10, están en peligro de extinción y 33 son vulnerables, lo que hace que dicha área tenga un alto valor en su diversidad biológica García (2010).

Principales características geológicas de la región

El Bloque Oriental Cubano presenta características geológicas muy particulares dentro del marco de la estructura geológica cubana. De ahí el gran interés que ha despertado en diversos investigadores, Quintas et al. (2002) y Iturralde-Vinent (1990) llevando a cabo estudios encaminados a profundizar en la estructura geológica cubana en general (Figura 2).

El cinturón plegado está constituido por terrenos oceánicos y continentales deformados y metamorfizados de edad pre-Eoceno Medio, representado por las unidades oceánicas, constituidas por las ofiolitas septentrionales.

Características tectónicas

El Bloque Oriental Cubano comprendido desde la falla Cauto-Nipe hasta el extremo oriental de la isla, presenta una tectónica caracterizada por su alta complejidad, dado por la ocurrencia de eventos de diferentes índoles que se han superpuesto en el tiempo y que han generados estructuras que se manifiestan con variada intensidad e indicios en la superficie (Rodríguez, 1998).

\section{Características Hidrogeológicas}

Debido al régimen de precipitaciones, particularidades hidrogeológicas regionales, características de las rocas acuíferas y parámetros hidrogeológicos existentes en el territorio, lo identificamos como una zona de elevada complejidad hidrogeológica. Se ha establecido para la región, la existencia de cinco complejos acuíferos fundamentales, a partir de la caracterización del tipo de rocas presentes, así como, de su capacidad para el almacenamiento en mayor o menor grado de aguas subterráneas. 


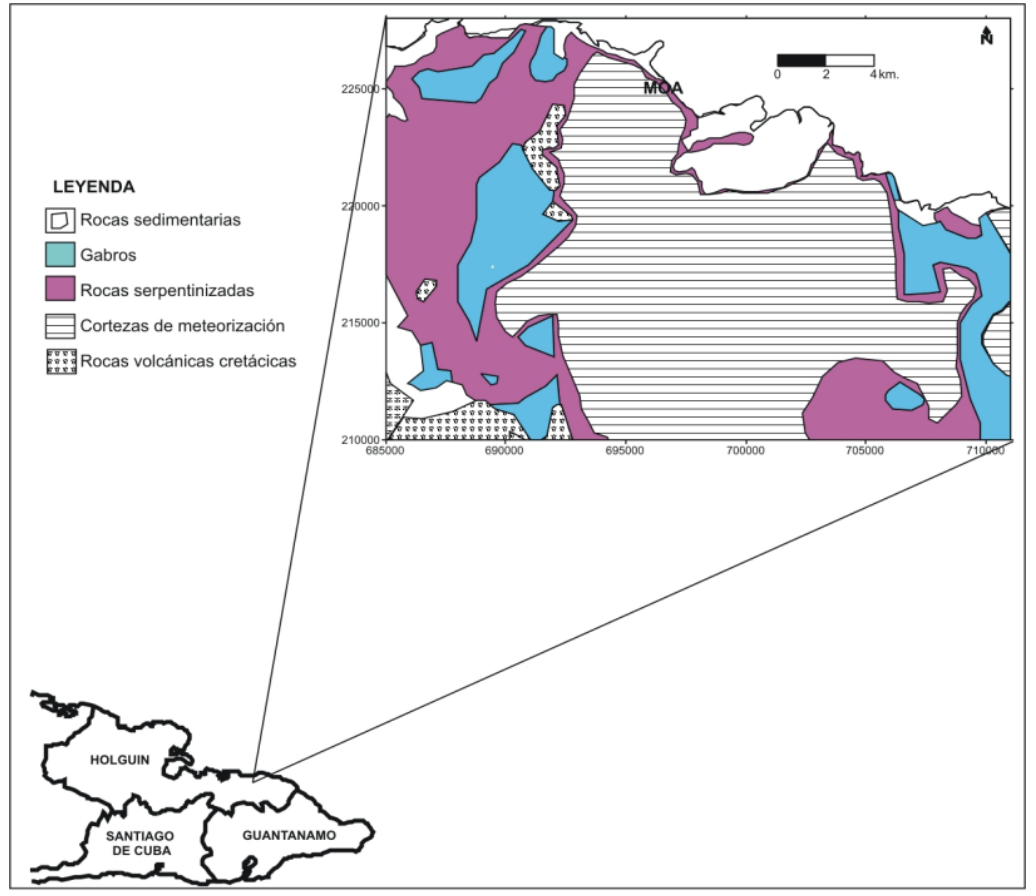

Figura 2: Mapa Geológico de la zona de estudio

Sismicidad

Por la posición geólogo-estructural que tiene el sector Calentura-Farallones, de estar bordeada por tres zonas sismogeneradoras coincidentes con fallas profundas que constituyen límites entre o interplacas, lo ubican dentro del contexto sismotectónico de Cuba Oriental (Oliva et al., 1989). Estas tres zonas son:

- Zona sismogeneradora Oriente: Está asociada a la falla transcurrente Bartlett-Caimán de dirección este-oeste. Constituye el límite entre la placa Norteamericana y Caribeña. A esta zona corresponde la más alta sismicidad de toda Cuba y con ella se encuentran asociados los terremotos de mayor intensidad con epicentros en el archipiélago cubano. La intensidad máxima pronóstico promedio para la zona es de VIII grados en la escala MSK, llegando hasta IX en el sector Santiago-Guantánamo. La magnitud máxima es de 8 grados en la escala Richter.

- Zona sismogeneradora Cauto-Nipe: Está asociada a la zona de fractura de igual nombre, con dirección suroeste-noreste desde las inmediaciones de Niquero hasta la bahía de Nipe. Constituye un límite ínter placa, que separa al Bloque Oriental Cubano del resto de la isla. La potencialidad sísmica de esta zona alcanza los 7 grados en la escala Richter, mientras que la intensidad sísmica, según el mapa complejo de la Región Oriental de Cuba señala valores entre VI y VII grados MSK.

\section{Situación socio- económica del territorio}

Económicamente Moa se encuentra dentro de uno de los municipios más desarrollados del país, dado por sus características socioeconómicas mixtas agroindustriales. El territorio se distingue por tener una estructura polarizada en la minería y la metalurgia no ferrosa, con signos de participación en la rama química. 


\subsection{Metodología para la evaluación de los impactos ambientales en las obras protectoras}

\section{Método para la selección de la metodología}

La selección del método de la metodología se fundamentó en las necesidades específicas de la obra y se tuvo en cuenta los criterios de Salomón (1997), un clásico de la evaluación de impacto, que plantea que las técnicas para evaluar impactos deben ser:

Comprensibles: Significa que deberán definir los diferentes impactos de la obra sobre el medio abiótico, biótico y socio-económico.

Dinámica: Al ser capaz de incluir variables adicionales e incorporar mediciones y técnicas predictivas.

Objetiva: Para analizar de forma imparcial todos los impactos.

Flexible: Para responder a variaciones del proyecto.

Implementable: No debe ser compleja para su implementación dentro del tiempo y los recursos disponibles.

Reproducibles: Significa que los resultados obtenidos deben proveer un sistema, que pueda ser aplicable en otros escenarios similares.

\subsection{Metodología para la evaluación de los impactos ambientales en las obras protectoras}

Para la identificación, caracterización y evaluación de los impactos, se utilizó la Metodología para la Realización de las Solicitudes de Licencia Ambiental y los Estudios de Impacto Ambiental, propuestas por el Ministerio de Ciencia Tecnología y Medio Ambiente y el Centro de Inspección y Control Ambiental (CICA) de Cuba, en su versión vigente del 2010, adaptadas a las condiciones específicas del objeto de investigación y la referencia del trabajo de evaluación de impactos de (García, 2010) y su ponderación (Tabla 1), Utilizando la observación directa, consultas de expertos, escenarios comparados, tormentas de ideas, las encuestas y entrevistas. Se procede asignarle un valor a cada impacto, en dependencia de los valores asignados según la Fórmula (1)

El impacto total se calculó según la expresión (1)

$$
\mathrm{I} T=\mathrm{Mg}+\mathrm{I}+\mathrm{S}+\mathrm{R}+\mathrm{O}+\mathrm{D}+\mathrm{C}
$$

Donde: Mg Magnitud, I Importancia, S Sinergismo, R Reversibilidad, O Ocurrencia, D Duración, C Certeza. Estos indicadores alcanzan un valor máximo de 10 puntos que luego se multiplicará por los siete indicadores para un total de 70 puntos total, que finalmente se promedia para ubicarlos en cada una de las categorías propuestas en términos de: compatibles, moderados, severos y críticos. 
Tabla 1: Ponderación de los Impactos.

\begin{tabular}{|c|c|}
\hline Naturaleza (Na) & Positivo (+) / Negativo (-) \\
\hline \multirow{3}{*}{ Magnitud (Mg) } & Leve: 1,2,3 \\
\hline & Moderado: 4.5,6 \\
\hline & Fuerte:7, 8,9,10 \\
\hline \multirow{4}{*}{ Importancia (I) } & Local: 1,2,3 \\
\hline & Zonal: 3,4,5 \\
\hline & Regional: 6,7,8 \\
\hline & Global: 9,10 \\
\hline \multirow{4}{*}{ Sinergismo (S) } & Sin sinergias aparentes: 1 \\
\hline & Con sinergias poco importantes: $2,3,4$ \\
\hline & Con sinergias importantes: 5,6,7 \\
\hline & Con sinergia catastrófica: $8,9,10$ \\
\hline \multirow{3}{*}{ Reversibilidad (R) } & Reversible: 1,2 \\
\hline & Reversible con medidas: $3,4,5,6,7$ \\
\hline & Irreversible: $8,9,10$ \\
\hline \multirow{3}{*}{ Ocurrencia (O) } & Directos o primarios: $1,2,3$ \\
\hline & Indirectos o secundarios: $4,5,6$ \\
\hline & Acumulativos: $7,8,9,10$ \\
\hline \multirow{3}{*}{ Duración (D) } & Corto plazo: $1,2,3$ \\
\hline & Mediano plazo: $34,5,6$ \\
\hline & Largo plazo: 7,8,9,10 \\
\hline \multirow{3}{*}{ Certeza (C) } & Poco probable: $1,2,3$ \\
\hline & Probable: $4,5,6$ \\
\hline & Inevitable: 7,8,9,10 \\
\hline Impacto Total (IT) 7 & 70 \\
\hline
\end{tabular}

La evaluación del impacto total tiene como finalidad llegar a clasificar los impactos ambientales en términos de:

Tabla 2: Escala de clasificación de los impactos del CITMA (2008).

\begin{tabular}{ll}
\hline RANGO & CLASIFICACIÓN \\
\hline IT $<18$ & IMPACTO COMPATIBLE \\
\hline IT $18-29$ & IMPACTO MODERADO \\
\hline IT $30-35$ & IMPACTO SEVERO/ MUY BENÉFICO \\
\hline IT $>35$ & IMPACTO CRÍTICO/ EXTREMADAMENTE BENÉFICO \\
\hline
\end{tabular}


Impacto Compatible: Son los que tienen muy poca entidad, si su efecto es perjudicial al cesar las causas que lo producen, en poco tiempo se restablecen las condiciones medio ambientales, originales, con el concurso de los procesos regenadores. Se clasifican en leve, local, directo, primario, reversible, sin sinergias, se recupera a corto plazo, poco probable y presenta un valor menor que 18 de impacto total.

Impacto moderado: Produce daños de poca magnitud, pero su importancia comienza a ser considerable. Tras el mismo las condiciones físicas originales se restablecen con el solo concurso de los mecanismos naturales del medio, aunque la recuperación es larga. Se clasifica en leve, regional en cierto grado, primario, reversible con dificultad, sin sinergias aparentes, se recupera a mediano plazo y es probable, alcanza un valor de 18-29 de impacto total.

Impacto severo: Se trata de impactos de magnitud notable y de gran importancia, cuando cesa la causa que lo origina, la recuperación de las condiciones iniciales del medio, se hace muy difícil y requiere a veces de la adopción y puesta en prácticas de medidas correctoras de sus efectos. Se clasifica con moderada intensidad, regional, indirecto o secundario, reversible con dificultad y medidas correctoras, con algunas sinergias, poco importantes, muy probable que se produzca, alcanza un valor de impacto total entre 30 y 35.

Impacto crítico: Es el impacto que por su enorme magnitud e importancia, supera el denominado Umbral del Impacto, que es el límite a partir del cual se considera que el deterioro el irreversible, la acción capaza de producirlo provoca la destrucción completa de elementos o recursos naturales que son piezas claves en el funcionamiento del paisaje, imponiendo en su lugar una dinámica regresiva continuamente adversa al establecimiento de las condiciones que posibilitarán su restitución. Se clasifica en fuerte, global o regional, acumulativo, irreversible, a largo plazo se mantiene, con sinergias importantes o catastróficas, inevitable y el impacto total alcanza un valor mayor que 35 .

\subsection{Propuestas de un Plan de medidas de mitigación y corrección de los impactos negativos}

Esta fase se considera la más importante, en ella se elabora un conjunto de medidas preventivas, correctoras y de mitigación que pueden generalizarse para atenuar los efectos negativos de la construcción de las obras protectoras. Estas medidas se relacionan con plazos establecidos en función del menor tiempo de permanencia de los efectos negativos sobre los factores del medio afectado y se agrupan en:

Medidas a corto plazo: Se aplicarán inmediatamente después de presentarse el impacto.

Medidas a mediano plazo: Serán aplicadas en el transcurso de la ejecución de la obra.

Medidas a largo plazo: Se ejecutarán en un período mayor de tiempo y serán fundamentalmente de mantenimiento. 


\section{RESULTADOS Y DISCUSIÓN}

\subsection{Acciones impactantes en las obras protectoras A, B y C}

Las acciones impactantes están relacionadas con las actividades que se realizan en la construcción de las tres obras protectoras denominadas A, B y C.

Están enmarcadas en las siguientes operaciones:

\section{Preparación del terreno}

a) Trabajo Topográfico

b) Eliminación de los obstáculos no útiles

c) Movimiento de equipos de transporte

d) Transportación

\section{Arranque de la roca}

e) Voladura (A) Utilización de martillos rompedores (B y C)

f) Extracción de la roca

g) Acarreo de escombros

3. Transportación de la roca

h) Traslado del material no útil

i) Movimiento de equipos de transporte.

j) Saneamiento del frente de trabajo.

4. Ventilación del túnel

k) Exploración

l) Perforación de pozos

m) Colocación de tuberías de ventilación

5. Fortificación

n) Laboreo de excavaciones

o) Construcción de cimientos, paredes y bóvedas

6. Operaciones auxiliares

p) Instalación eléctrica.

q) Construcción de desagües.

r) Trabajos Topográficos.

s) Colocación de tuberías y cables.

\section{Acondicionamiento}

t) Crear condiciones indispensables para la estancia del personal (A). 


\subsection{Resultados de los factores ambientales impactados en las obras protectoras A, B y C}

En las encuestas realizadas a los pobladores, expertos y la observación directa. Pudimos constatar que los factores ambientales identificados alcanzan en su totalidad más del $70 \%$ de los criterios, considerándolos que son los que más impactos reciben producto de las acciones que se llevan a cabo en la construcción de las obras protectoras.

Los factores ambientales identificados en las (Obras A, B, y C) son los siguientes:

\section{Medio físico}

- Suelo

- Agua

- Atmósfera

Medio biótico

- Vegetación

- Flora

- Fauna

Medio sociocultural

- Población

- Economía

Medio perceptual

- Paisaje

\subsection{Impacto ambiental en las obras (A, B, C)}

A través de las consultas a expertos de las encuestas, entrevistas a vecinos y trabajadores de las obras, se identificaron los siguientes impactos producidos sobre los factores ambientales que a continuación se relacionan.

\section{Al suelo}

1- Pérdida y alteración de la capa del suelo fértil en los emboquilles. A, B y C. (Figura 3)

2- Cambios de las propiedades físico- químicas de los suelos. (A, B, C)

3- Compactación crítica de los suelos. (B, C)

4- Afectación del estado tensional de macizo. (C)

Debido al transporte de la roca y a las labores de excavación de las entradas y salidas del túnel (calicatas) y los pozos de ventilación y al movimiento de equipos. 


\section{Al agua}

5- Cambios de las propiedades químicas. $(A, B, C)$

6- Contaminación del acuífero. $(A, B, C)$

7- Desvío del agua subterránea. $(A, B, C)$

\section{A la Atmósfera}

8- Disminución de la calidad atmosférica por emisiones de gases, polvo. (A, B, C)

9- Incremento en el nivel de ruidos. $(A, B, C)$

\section{A la vegetación}

10- Pérdida de la vegetación. (A, B, C) (Figura 4)

\section{A la flora}

11- Pérdida de especie de plantas exóticas. $(A, B, C)$

\section{A la fauna}

12- Alteración del hábitat de especies de reptiles e insectos. $(A, B, C)$

\section{A la población y economía}

13- Generación de nuevas fuentes de empleo. (A, B, C)

14- Aumento de riesgos de accidentes de trabajo. (A, B, C)

15- Incremento del potencial defensivo de la región. (A, B, C)

16- Proliferación de vectores. $(A, B, C)$

17- Deterioro a las edificaciones. $(A)$

18- Rotura del pavimento (A, B, C)

\section{Al paisaje}

19 - Modificación de las características visuales del paisaje. (A, B, C)

20 - Disminución del atractivo paisajístico. $(B, C)$

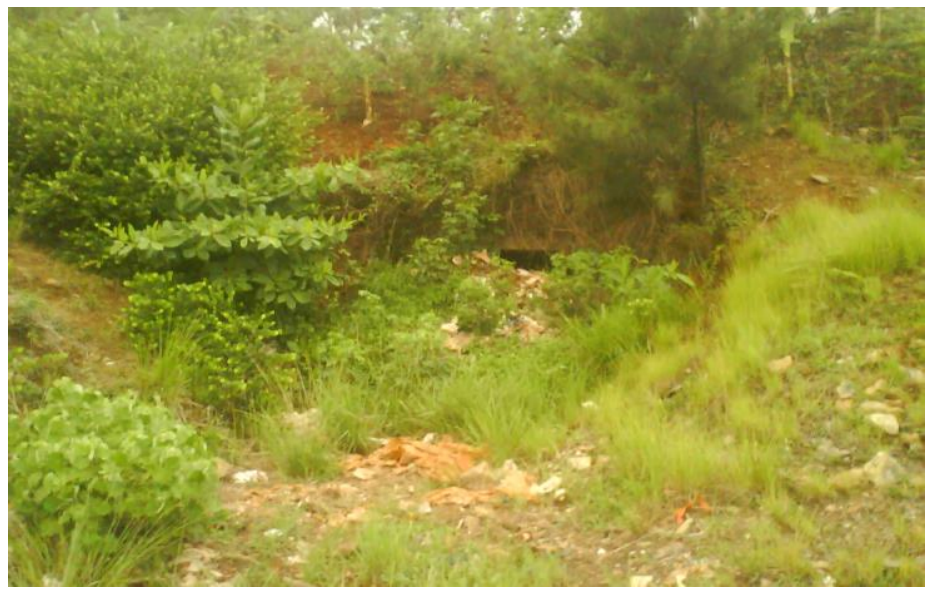

Figura 3: Pérdida y alteración de la capa del suelo fértil en el emboquille de la obra. 


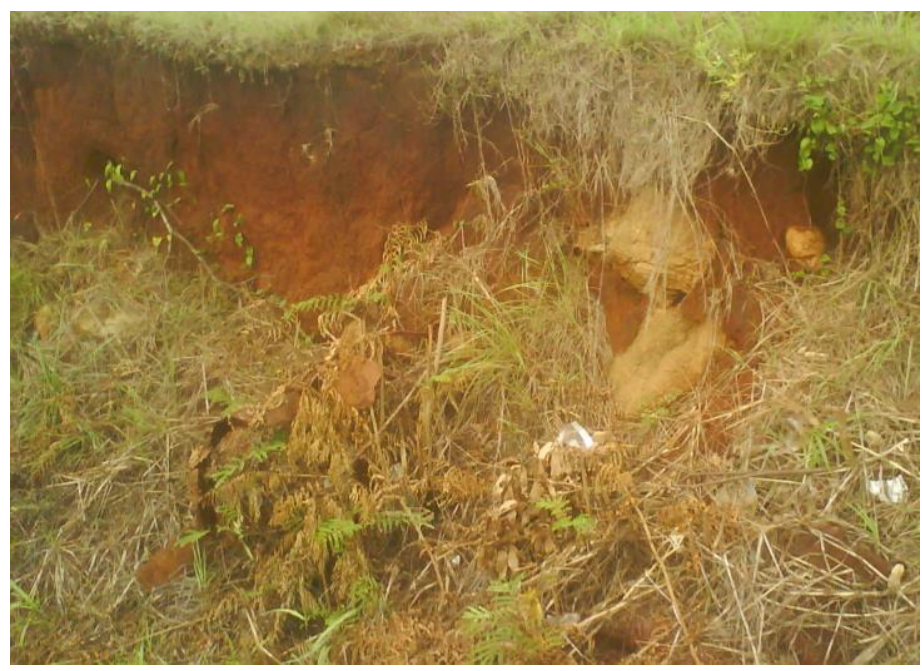

Figura 4: Pérdida de la vegetación en los alrededores de la obra.

Fundamentalmente en las obras protectoras que están paralizados e inhabitables por lo que hay permanencia de huecos y durante las etapas de preparación al terreno, transporte de la roca y la ventilación producto de la emanada de polvo que ahí se produce.

Después de la determinación de los impactos producidos sobre los factores ambientales se realiza la valoración de cada impacto teniendo en cuenta los indicadores propuestos por el CITMA 2010.

4.4. Análisis y ponderación de los impactos

Tabla 3: Matriz de valoración de los impactos ambientales según CITMA 2010.

\begin{tabular}{|c|c|c|c|c|c|c|c|c|c|c|}
\hline \multirow[t]{2}{*}{ Impactos } & \multicolumn{8}{|c|}{ Indicadores } & \multirow[t]{2}{*}{$\begin{array}{l}\text { Ponderación de } \\
\text { los Impactos }\end{array}$} & \multirow[t]{2}{*}{ IT } \\
\hline & $\mathrm{Na}$ & $\mathrm{Mg}$ & I & $\mathbf{R}$ & D & C & 0 & $S$ & & \\
\hline 1 & - & 3 & 3 & 4 & 3 & 4 & 3 & 4 & 24 & $M$ \\
\hline 2 & - & 3 & 3 & 5 & 3 & 4 & 5 & 4 & 30 & $M$ \\
\hline 3 & - & 4 & 3 & 4 & 4 & 4 & 4 & 2 & 25 & $M$ \\
\hline 4 & - & 5 & 3 & 4 & 2 & 3 & 3 & 3 & 23 & $M$ \\
\hline 5 & - & 3 & 2 & 5 & 1 & 5 & 3 & 3 & 22 & $M$ \\
\hline 6 & - & 3 & 2 & 1 & 1 & 3 & 3 & 3 & 16 & \\
\hline
\end{tabular}




\begin{tabular}{|c|c|c|c|c|c|c|c|c|c|c|}
\hline & & & & & & & & & & C \\
\hline 7 & - & 1 & 1 & 1 & 1 & 1 & 2 & 3 & 10 & C \\
\hline 8 & - & 5 & 5 & 4 & 3 & 5 & 6 & 3 & 31 & s \\
\hline 9 & - & 1 & 2 & 3 & 5 & 5 & 3 & 1 & 19 & $M$ \\
\hline 10 & - & 5 & 2 & 5 & 1 & 5 & 2 & 1 & 21 & $M$ \\
\hline 11 & - & 3 & 2 & 5 & 3 & 5 & 2 & 3 & 23 & $M$ \\
\hline 12 & - & 3 & 2 & 5 & 3 & 5 & 2 & 3 & 23 & $M$ \\
\hline 13 & + & 3 & 3 & 1 & 5 & 5 & 3 & 3 & +23 & $M$ \\
\hline 14 & + & 6 & 3 & 2 & 3 & 8 & 3 & 7 & 32 & B \\
\hline 15 & - & 3 & 2 & 3 & 5 & 5 & 3 & 3 & +32 & IN \\
\hline 16 & - & 5 & 2 & 5 & 5 & 5 & 3 & 5 & 30 & $S$ \\
\hline 17 & - & 5 & 5 & 5 & 5 & 5 & 3 & 3 & 31 & $S$ \\
\hline 18 & - & 5 & 3 & 5 & 1 & 5 & 3 & 5 & 27 & $M$ \\
\hline 19 & - & 5 & 3 & 5 & 1 & 5 & 3 & 5 & 27 & $M$ \\
\hline 20 & - & 5 & 3 & 5 & 1 & 5 & 3 & 5 & 27 & $M$ \\
\hline
\end{tabular}

Del análisis de la valoración de cada uno de los impactos reflejado en la (tabla 2), teniendo en cuenta los indicadores propuestos en su ponderación. Se obtuvo un total de 21 impactos, de ellos solo dos se clasificaron como positivos (Aumento del potencial defensivo de la región y la generación de nuevas fuentes de empleo). El resto es de naturaleza negativa. Dentro de los impactos compatibles: La contaminación del acuífero y el desvío del agua subterránea. Lo que significa que tienen poca entidad y sus efectos son perjudiciales al cesar las causas que lo producen. Como impactos severos son: la disminución de la calidad atmosférica por emisiones de gases, polvo. Se trata de un impacto de magnitud notable y de gran importancia, el aumento de riesgos de accidentes de trabajo. Se trata de un impacto de magnitud notable y de gran importancia, el incremento del potencial defensivo de la región. Se considera muy benéfico teniendo en cuenta que la construcción de las obras y su unificación al sistema defensivo de la región, aumenta de forma considerable la protección de los recursos humanos, la proliferación 
de vectores. Se trata de un impacto negativo de magnitud notable y de gran importancia y el Deterioro de las edificaciones. Se trata de un impacto de magnitud notable y de gran importancia. Presente principalmente en la Obra A.

El resto de los impactos se consideran moderados, producen daños de moderada intensidad, son regional, indirectos, pero su importancia es considerable y debe tenerse en cuenta en los tres tipos de casos, la implementación de medidas preventivas, correctoras y de mitigación.

\subsection{Medidas de mejora de la situación constructiva de las Obras protectoras A, B, C}

Debido a la paralización temporal de las obras, se recomendó de manera inmediata ejecutar algunas acciones de carácter particular, para mejorar las condiciones que presentaban las obras $A, B$ y $C$ al inicio de la investigación, pero que ya en la actualidad se ha ejecutado.

Obra A: En el lado este, frente a la entrada, debe crearse un pequeño borde para contener las aguas exteriores y a partir de la misma pendiente de entrada comenzar una zanja de desagüe con pendiente mínima, buscando el primer escalón de la acera del edificio, para que a través de un tubo de $200 \mathrm{~mm}$ cuya cota invertida sea de $300 \mathrm{~mm}$ por debajo de la cota del piso, pase por debajo de la acera.

Obra B. Frente a la entrada de la obra debe eliminarse el material producto de las excavaciones y todo lo que se encuentra a su lado oeste, pues constituye una cortina de contención y vertimiento de las aguas pluviales hacia el interior de la obra. En el frente y en el lado este de la obra crear un pequeño talud de $50 \mathrm{~mm}$ de alto (al mismo borde de la obra) con el propósito de desviar las aguas que correrán pendiente abajo, y en la etapa de terminación reconstituir toda el área de entrada por donde en estos momentos, se evacuan los materiales, hasta dejar como entrada, solo una pequeña pendiente que debe ser techada.

Obra C. Frente a la entrada de este de la obra, en el lado este y a todo lo largo del frente, hasta conectarse con la otra boca, crear un borde de $50 \mathrm{~cm}$ de alto tratando de que éste abarque la menor área posible, dejando solo el espacio necesario para las labores de trabajo. Esta acción es correctora, ya que primeramente los tuneleros realizarán una obra de drenaje que abarcaba un área muy extensa, que provocaba, que al evacuarse las aguas de la parte exterior del túnel, se acumulara en el interior del mismo.

\subsection{Medidas generales de prevención, corrección y de mitigación de los impactos negativos de la construcción de túneles populares}

Como resultado del estudio detallado de los impactos más significativos en las Obras A, B y $C$, se elaboró un conjunto de medidas preventivas que pueden generalizarse para atenuar los efectos negativos de la construcción de las obras protectoras. Estas medidas se relacionan con plazos establecidos en función del menor tiempo de permanencia de los efectos negativos sobre los factores del medio afectado y se agrupan en: 


\section{Entre las medidas a corto plazo están:}

Reducir en lo posible la cantidad de área de suelo a afectar durante la apertura de las calicatas, de los pozos de ventilación, y donde se depositará el material extraído en zonas aledañas.

Durante la etapa de preparación de terreno, separar la capa de suelo vegetal y depositarla en zonas alejadas de los lugares donde se verterá el resto del material extraído, para ser utilizada en la recuperación de las áreas, una vez concluido a la obra.

Entregar al personal que laborará en la obra, los medios de protección individual necesarios para el desarrollo de las diferentes etapas constructivas, tales como; linterna, espejuelos, cascos y guantes, entre otros.

Eliminar de forma sistemática el material extraído, para evitar la posible interrupción del drenaje superficial y la proliferación de vectores.

Depositar el material extraído en el o los sitios previamente autorizados, principalmente en oquedades o terrenos que necesitan rellenos.

Realizar un estudio detallado de las filtraciones de agua que tienen lugar en el interior de estas obras, con el objetivo de diseñar un sistema para el drenaje de las mismas.

Chequear el cumplimiento de las medidas de seguridad y protección adoptadas luego de terminada la obra, para impedir actos delictivos.

Elaborar el estudio de estabilidad y factibilidad de cada obra con el fin de determinar con mayor exactitud su ubicación y uso futuro.

\section{Las medidas a mediano plazo que se recomiendan son:}

Repoblar los espacios afectados sin vegetación natural ni pasto para evitar la erosión del suelo y propiciar su enmascaramiento.

Establecer un programa de preparación ambiental para los trabajadores del proyecto de construcción de túneles populares con el objetivo de lograr la introducción de la dimensión ambiental en esta actividad.

Desarrollar un programa de Educación Ambiental y preparación de la población de los asentamientos, que involucra las organizaciones de masas el Ministerio de Educación y el Poder Popular con el objetivo de lograr que la población comprenda la importancia que presenta para su protección la utilización de los túneles populares como refugio en tiempo de guerra.

\section{A largo plazo se deben desarrollar las medidas siguientes:}

Cerrar herméticamente las bocas de los pozos de ventilación y realizar sistemáticamente el control de vectores (mosquitos y roedores) en los mismos después de terminada la obra.

Teniendo en cuenta que la geología de las áreas no es favorable desde el punto de vista de sostenimiento y seguridad estructural de la obra, se deben realizar los estudios correspondientes para el cálculo de la fortificación en cada caso y cumplir los lineamientos generales para la construcción de este tipo de obra.

\subsubsection{Programa de información general para la población}


La elaboración de un programa de información general forma parte del plan de medidas recomendadas y que deberán ser ejecutadas en todas las fases del proyecto para la población, (según la programación del proyecto CITMA aprobado), que establece la participación de los organismos de salud y educación, las organizaciones de masas y el Poder Popular y las responsabilidad de cada uno en el cronograma de ejecución.

A la vista de la situación actual de amenazas, este programa se convierte en un instrumento estratégico de singular importancia que puede proporcionar a la población en general la información necesaria para comprender el riesgo de no protegerse y las ventajas de contar con las instalaciones de protección apropiadas. Este de forma general debe promover la generación, desarrollo y consolidación de la cultura necesaria de protección en caso de guerra y la auto preparación individual y colectiva.

Las acciones más importantes de este programa se relacionan a continuación:

- Impartir en cada obra, conferencias al personal que trabaja en las mismas sobre:

- La necesidad de desarrollar las obras según el proyecto de construcción.

- Las afectaciones que provoca la construcción de túneles populares sobre el medio.

- La necesidad de simultanear las acciones del proyecto con las medidas correctoras correspondientes.

- Divulgar el plan de medidas de prevención, corrección y de mitigación de impactos negativos, a través de murales, carteles, conferencias, seminarios, plegables.

- Realizar talleres con los ejecutores para analizar los resultados de la aplicación de las medidas de mitigación registradas en el proyecto.

- Establecer una estrategia con la participación de todos los actores identificados, encaminada a lograr la aceptación de la población en el uso de estas construcciones como obras de protección en tiempo de guerra, que recoja acciones educativas preventivas.

- Implementar murales, carteles, conferencias, seminarios, plegables, para sensibilizar a los trabajadores de las obras, y la población en general sobre la importancia del uso de los túneles populares para la protección en tiempo de guerra.

- Divulgar los beneficios de las instalaciones de protección a través de charlas, intercambios, plegables etc.

- Alertar, para convencer a la población, sobre los daños que pueden sufrir en caso de ataques del enemigo, las personas no protegidas.

- Diseñar y coordinar materiales que apoyen la divulgación de conocimientos que promuevan las conductas de autoprotección (carteles, folletos, críticos, guías prácticas, materiales audiovisuales).

- Vincular a la población con el acondicionamiento y mantenimiento de las obras de protección de su área.

- Incorporar materias con perspectivas de protección civil en todos los niveles educacionales. 


\section{CONCLUSIONES}

Se identificaron un total de 21 impactos, de ellos solo dos son de naturaleza positiva (Aumento del potencial defensivo de la región y la generación de nuevas fuentes de empleo). El resto es de naturaleza negativa que representa un 90,7\%. Los impactos negativos clasificados como severos alcanzan un valor entre 30 y 35 son: la disminución de la calidad atmosférica por emisiones de gases, polvo, el aumento de riesgos de accidentes de trabajo, la proliferación de vectores, el deterioro de las edificaciones. Se trata de impactos de magnitud notable y de gran importancia por los efectos que producen al medio y el muy benéfico está dado por el incremento del potencial defensivo de la región. Reciben un total de impactos negativos alto sobre los factores ambientales, la población, la economía, la atmósfera, el suelo y el paisaje. Las actividades que sus acciones producen un total de impactos negativos alto son la preparación del terreno, el arranque de la roca y la transportación de la roca. Las medidas preventivas, correctoras y de mitigación en los plazos establecidos en el estudio, satisfacen las necesidades que la problemática ambiental demanda generados por la construcción de túneles populares y su eficiencia dependerá de su correcta implementación. La evaluación ambiental de la construcción de las obras protectoras, permitió identificar, caracterizar y evaluar los impactos producidos, con un alto nivel de generación de impactos negativos sobre los componentes ambientales.

\section{REFERENCIAS}

Aguilera F (2003). Estudio del Impacto Ambiental Ocasionado por la Explotación del yacimiento fluvial de Arena y Grava "Río Nibujón". Tesis de Maestría. Instituto Superior Minero Metalúrgico de Moa, 91p.

Almaguer Y (2013). Evaluación de la susceptibilidad del terreno a la rotura por desarrollo de deslizamientos en el yacimiento Punta Gorda. Tesis Doctoral. Instituto Superior Minero Metalúrgico de Moa, 95p.

Cabrera M (1987). Evaluación de Impacto Ambiental. Revista de Obras públicas. 134 (3263): 737$743 p$.

Cartaya M, Blanco, R (1997). Informes Ingeniero - Geológicos y valoración de estabilidad de los túneles populares del municipio Moa. Estado Mayor Municipal de la Defensa Civil - Moa. 100 p.

Cartaya M (2000). Caracterización geomecánica de los macizos rocosos de algunas minas y túneles subterráneos de la región oriental del país: Memorias "Primer Simposio Internacional la geodesia y la Geomecánica Aplicadas a la Construcción: Ciudad de la Habana. Cuba. 122$130 p$.

Conesa V (1997). Guía metodológica para la evaluación de impacto ambiental 2da edición Madrid Editorial Madrid Prensa. Madrid. 1997. 3a edición. Referencia de la biblioteca de Filosofía: FL/ TD 194.6.C66. 108p.

García C (2010). Evaluación de impacto ambiental de la construcción de túneles populares en el municipio Moa. Tesis de Grado. Instituto Superior Minero Metalúrgico de Moa, 69p. 
Gómez D (1999). Evaluación del Impacto Ambiental. Ed. Mundi-Prensa y Editorial Agrícola Española, S.A. Madrid. 1a edición. Referencia de la biblioteca de Filosofía: FL/TD 194.4. G6.100p

Iturralde-Vinent M (1990) Las ofiolitas en la constitución geológica de Cuba. Ciencias de la Tierra y del Espacio, 17, 8-26p.

Hernández T (2013). Estudio de Impacto Ambiental del Proyecto de Explotación de loa años 2002-2005 del Yacimiento Punta Gorda. Tesis de Maestría. Instituto Superior Minero Metalúrgico de Moa, 104p.

Hurtado G (2012). Estudio de la influencia en el medio ambiente del sistema de generación de la empresa del níquel Comandante Ernesto Che Guevara de Moa y el Sistema de Transmisión Eléctrico adyacente. Tesis doctoral ISMMM. 86p.

Leopold, L. B. et al., (1971). A Procedure for Evaluating Environmental Impact. United States Geological Survey, Geological Survey Circular No. 645, Washington, D.C.13p.

Ministerio de Obras Públicas, Transporte y Medio Ambiente. (1995). Guía para la Elaboración de Estudios del Medio Físico, Contenido y Metodología. Serie Monografías. España.

Ministerio de Ciencia, Tecnología y Medio Ambiente. Resolución No.58 (1998). Regulaciones generales para la protección del medio ambiente y los recursos naturales en situaciones excepcionales.

Ministerio de Ciencia Tecnología y Medio Ambiente. (1999). Resolución 77/99 del CITMA. Reglamento del Proceso de Evaluación de Impacto Ambiental.

Ministerio de Ciencia Tecnología y Medio Ambiente. Centro de Inspección y Control Ambiental. (2001).Guías para la Realización de las Solicitudes de Licencia Ambiental y los Estudios de Impacto Ambiental.

Noa M (2003). Indicaciones metodológicas para la elección del método de arranque de las rocas durante el laboreo de excavaciones subterráneas horizontales de pequeña y mediana sección en Cuba oriental. Tesis Doctoral. Instituto Superior Minero Metalúrgico.

Oliva G., Lluis E., Sánchez E. A., Hernández J. R., Propin E., Buznego E., Lorenzo A. C., Mon M. \& Azcue A. (1989) Nuevo atlas nacional de Cuba. Instituto de Geografía de la Academia de Ciencias de Cuba.

Quintas F., Almaguer Y., Rodríguez A., Rodés A., Vargas A., (2002) Cartografía geológica del basamento del yacimiento Punta Gorda a escala 1:2 000. Subprograma del proyecto de Modelación Geotecnológica de la Empresa Enerito Che Guevara. Departamento de geologia, ISMM, Cuba. 120 p.

Rodríguez A (1998). Estudio morfotectónico de Moa y áreas adyacentes para la evaluación de riesgos de génesis tectónica. ISMM, Departamento de Geología. Tesis Doctoral. 118 p.

Salomón C (1997). Water Resources Aseecsment Methodology (Wram)- Impact. Assessment and Alternative Evaluation.

Sánchez L (2011). Evaluación de Impacto Ambiental. Segundo Curso Internacional de Aspectos Geológicos de Protección Ambiental. 53-73p.

Suárez I (2007). Estudio de Impacto Ambiental en obras Subterráneas en funcionamiento del EO. Tesis de Maestría Instituto Superior Minero Metalúrgico de Moa.115p.

Sorenssen, J. C. Moss, M. L. (1973). Procedures and Programmes to Assist in the Environmental Impact State Ment Process. University of California. USA. 250p 